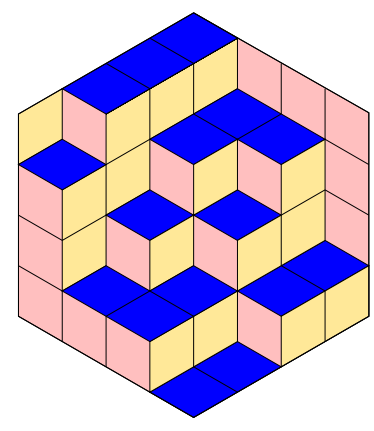

ALGEBRAIC COMBINATORICS

Ian Le

Intersection Pairings for Higher Laminations

Volume 4, issue 5 (2021), p. 823-841.

<http://alco.centre-mersenne.org/item/ALCO_2021_4_5_823_0>

(c) The journal and the authors, 2021.

Some rights reserved.

(c) BY This article is licensed under the

Creative Commons ATtribution 4.0 International LiCEnSE.

http://creativecommons.org/licenses/by/4.0/

Access to articles published by the journal Algebraic Combinatorics on the website http://alco.centre-mersenne.org/ implies agreement with the Terms of Use (http://alco.centre-mersenne.org/legal/).

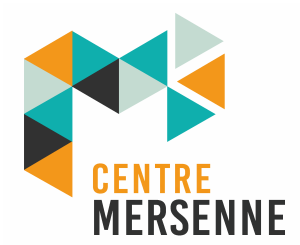

Algebraic Combinatorics is member of the Centre Mersenne for Open Scientific Publishing www.centre-mersenne.org 


\title{
Intersection Pairings for Higher Laminations
}

\author{
Ian Le
}

\begin{abstract}
One can realize higher laminations as positive configurations of points in the affine building [7]. The duality pairings of Fock and Goncharov [1] give pairings between higher laminations for two Langlands dual groups $G$ and $G^{\vee}$. These pairings are a generalization of the intersection pairing between measured laminations on a topological surface.

We give a geometric interpretation of these intersection pairings in a wide variety of cases. In particular, we show that they can be computed as the minimal weighted length of a network in the building. Thus we relate the intersection pairings to the metric structure of the affine building. This proves several of the conjectures from [9]. We also suggest the next steps toward giving geometric interpretations of intersection pairings in general.

The key tools are linearized versions of well-known classical results from combinatorics, like Hall's marriage lemma, König's theorem, and the Kuhn-Munkres algorithm, which are interesting in themselves.
\end{abstract}

\section{INTRODUCTION}

This paper has two goals. The first goal is to give geometric interpretations of the intersection pairings between higher laminations. For simplicity, let us restrict attention to higher laminations on a disc. In this case, higher laminations arise as the tropical points of configuration spaces of flags. In [7], we showed that higher laminations on a disc are given by configurations of points in the affine building. We will later define the affine building, but for now, the only relevant fact is that it is a metric space that behaves in many ways like a symmetric space.

In [1] and subsequent works, Fock and Goncharov give a series of conjectures about the properties that such a pairing should satisfy. The existence and construction of a pairing satisfying these properties was not known until the work of Gross, Hacking, Keel and Kontsevich [5]. The pairing is defined in [5] in a complicated, highly recursive way. It is natural to ask whether this pairing can be defined using the geometry of affine buildings. In this paper, we give such a geometric interpretation in a wide variety of cases. We show that the intersection pairing is given by the minimal weighted length of a network in the building. This suggests that such a geometric interpretation should exist in general, and we explain some conjectures about this at the end of the paper.

Let us also note that many combinatorial questions in Lie theory (canonical bases, structure constants, tensor multiplicities) are equivalent to counting problems for higher laminations.

Manuscript received 10th September 2017, revised and accepted 6th May 2021.

KEYWORDS. Discrete geometry, buildings, matroid, convexity, tropical geometry, cluster algebras. 
Our strategy is as follows. The properties of the pairing, conjectured by Fock and Goncharov and proved in [5], reduce the computation of the pairing to the computation of certain invariants of lattices that we call $f_{i j k}^{t}$. The main theorem, Theorem 5.4, gives a metric interpretation of the function $f_{i j k}^{t}$. This metric interpretation is computationally considerably simpler than the original definition, and Theorem 4.3 actually gives an algorithm for computing the function using the metric interpretation. We start by proving Theorem 4.3, then use it to deduce Theorem 5.4. After that, we explain how it can be used to calculate the intersection pairing in many cases.

Our second goal is to argue that Theorem 4.3 is interesting in itself, and may be of interest to combinatorialists, particularly those studying matroid theory. The proof of Theorem 4.3 involves linearizations of well-known theorems of combinatorics of Hall, König and Kuhn-Munkres. We expect that conjectural generalizations of Theorem 4.3 discussed in Section 6 will also be linearizations of combinatorial statements of minmax type. It is an interesting question to ask which statements of min-max type have linear generalizations. Generalizations of this type may be related to min-max statements in the theory of valuated matroids [11].

Moreover, Theorem 4.3 is valid over any discrete valuation ring. We wonder whether it may be useful to number theorists who use buildings in their work.

Finally, we say something about the notational conventions of this paper. Theorem 5.4 is essentially a reformulation of Theorem 4.3. The latter statement deals with some other invariants of lattices, which we call $c$ and $A$, which differ from $f_{i j k}^{t}$ by a sign and some normalizations (see Remark 5.3). We use two sets of notation for several reasons. First, we want to remind the reader of the subtle differences in sign and normalization. Second, it turns out that whereas the functions $c$ and $A$ are welladapted to the proof of Theorem 4.3, they are not as natural from the point of view of the geometry of the affine building. Finally, the notations $c$ and $A$ were chosen for their similarity with the analogous quantities used in the Kuhn-Munkres theorem, of which Theorem 4.3 is generalization.

We now summarize the contents of this paper. In Section 2, we review the theorems of Hall, König and Kuhn-Munkres. In Section 3, we will discuss linear generalizations of Hall's and König's theorems. In Section 4, we will introduce and prove a linearized version of the Kuhn-Munkres theorem. In Section 5, we will define higher laminations, and describe the intersection pairings and show how our generalization of the KuhnMunkres theorem can be applied to give an interpretation of intersection pairings of higher laminations in terms of the metric geometry of the affine building. In Section 6, we discuss generalizations and the next non-trivial case of intersection pairings for which we have only a conjectural description of the intersection pairing.

\section{HALL'S THEOREM AND ITS GENERALIZATIONS}

We begin by recalling some classical theorems from combinatorics: Theorems 2.1, 2.2 and 2.3. These theorems have linear generalizations, which give Theorem 3.1, Proposition 3.2 and Theorem 4.3, respectively. Proposition 3.2, which is a minor extension of Theorem 3.1, will be used in the proof of Theorem 4.3 (in the same way that Theorem 2.2, a minor extension of the more well-known Theorem 2.1, may be used to prove Theorem 2.3). We aim to stress the analogies between the combinatorial statements and their linear generalizations.

Let us recall the marriage problem. Consider the sets $S_{1}, S_{2}, \ldots, S_{r}$. The marriage problem asks whether one can find elements $x_{i}$ in each of the sets $S_{i}$ such that $x_{i} \neq x_{j}$ for $i \neq j$. In this situation, $x_{1}, \ldots, x_{r}$ will be called a system of distinct representatives for the sets $S_{i}$. 
Hall's theorem or Hall's Marriage Lemma gives a necessary and sufficient condition for a system of distinct representatives to exist:

TheOrem 2.1 (Hall). Let $S_{1}, S_{2}, \ldots, S_{r}$ be sets. A system of distinct representatives of the sets $S_{i}$ exists if and only if

$$
\left|\bigcup_{i \in I} S_{i}\right| \geqslant|I|
$$

for each subset $I \subseteq[r]$.

Hall's theorem can be phrased in terms of bipartite graphs. Let us imagine our bipartite graph with vertices divided into two sets, one set of vertices on the left and one set of vertices on the right. On the left, we may put $r$ vertices and label them $1, \ldots, r$. On the right, we put all elements of the sets $S_{i}$. We can then draw edges connecting each vertex $i$ on the left to all the elements in $S_{i}$. Then finding a system of distinct representatives is the same as finding a matching in this graph using all the vertices $1, \ldots, r$.

A refinement of Hall's theorem is König's theorem. Instead of giving conditions for a matching using all $r$ vertices to exist, it provides a formula for the maximum size of a matching:

Theorem 2.2 (König). Let $S_{1}, S_{2}, \ldots, S_{r}$ be sets. The maximum number of distinct representatives of the sets $S_{i}$ is

$$
\min _{I \subseteq[r]}\left(\left|\cup_{i \in I} S_{i}\right|+r-|I|\right) .
$$

As was the case with Hall's theorem, this can be interpreted in terms of bipartite graphs. Again suppose we have $r$ vertices on the left representing the sets $S_{i}$, and vertices on the right representing the elements of the sets $S_{i}$. Suppose that $I \subset[r]$ is chosen to minimize $\left(\left|\cup_{i \in I} S_{i}\right|+r-|I|\right)$. Then we can find a vertex cover of the graph (a set of vertices of the graph such that each edge is incident to at least one of these vertices): take all the vertices corresponding to the elements in $\cup_{i \in I} S_{i}$ and all the vertices in $[r] \backslash I$. This is a vertex cover of size $\left|\cup_{i \in I} S_{i}\right|+r-|I|$. It is clear that any vertex cover must be larger than the size of any matching. König's theorem says that the maximal matching has the same size as the minimal vertex cover. Note that if we fix a minimal vertex cover, any maximal matching must use each vertex of the minimal cover exactly once. (Also, if we fix a maximal matching, any minimal cover must cover exactly one of the vertices of each edge in the matching.)

Finally, König's theorem can be used to prove a theorem of Kuhn and Munkres. Let $\left[c_{i j}\right]$ be an $n \times n$ real matrix. A transversal of $\left[c_{i j}\right]$ is a choice of $n$ entries of the matrix, one entry in each row and each column. There are $n$ ! such transversals. For each transversal, we can consider the sum over that transversal. We would like to find the maximal possible value for this sum.

Suppose we have some real numbers $a_{i}$ and $b_{j}$ for $1 \leqslant i, j \leqslant n$ such that $a_{i}+b_{j} \geqslant c_{i j}$. We can call the set of real numbers $a_{i}$ and $b_{j}$ a potential. It is clear that the sum of any transversal is less than or equal to $\sum_{i} a_{i}+\sum_{j} b_{j}$. Thus the sum of any transversal is less than the sum of any potential. Then we have the following theorem:

Theorem 2.3 (Kuhn and Munkres). Let $\left[c_{i j}\right]$ be a real $n \times n$ matrix. Then the maximal sum of a transversal of $\left[c_{i j}\right]$ equals the minimal sum $\sum_{i} a_{i}+\sum_{j} b_{j}$, where we require that for all $i, j, a_{i}+b_{j} \geqslant c_{i j}$. Moreover, if the $c_{i j}$ are integers, the a's and b's can be taken to be integral as well.

In fact, the theorem above is actually an algorithm for constructing both the transversal and the potential. Once we find a transversal that is equal to the sum 
of the potential, then we know we have found both the maximal transversal and the minimal potential. Alternatively, we may interpret this as saying that the potential witnesses the fact that we have found a maximal potential.

REMARK 2.4. Let us point out that the algorithm for constructing the transversal and the potential is much more efficient than computing all $n$ ! transversals.

Replacing $\left[c_{i j}\right]$ by $\left[-c_{i j}\right]$ we can prove a similar theorem that the minimal transversal is equal to the maximal sum of a potential $a_{i}$ and $b_{j}$ where $a_{i}+b_{j} \leqslant c_{i j}$.

Note that in the theorems of both König and Kuhn-Munkres, we have that the maximum of one quantity is equal to the minimum of some other quantity. The linear generalizations which we will consider will also share this feature.

\section{Linearization}

We will need a linearization of Hall's marriage theorem. This theorem is a specialization of Rado's theorem on matroids, and was rediscovered by Moshonkin [12], [10]:

TheOREM 3.1 (Rado). Let $W_{1}, W_{2}, \ldots, W_{r}$ be subspaces of an ambient vector space $V$. Then a system of linearly independent representatives of the $W_{i}$ exists if and only if for each subset $I \subseteq[r]$,

$$
\operatorname{dim} \sum_{i \in I} W_{i} \geqslant|I| .
$$

Here $\sum_{i \in I} W_{i}$ is the sum of the vector subspaces $W_{i}$. As in Hall's marriage theorem, the condition is clearly necessary, and it turns out to be sufficient.

One can imagine a bipartite graph with the set $[r]$ on the left, and all the vectors in the vector space $V$ on the right. We connect a vertex $i$ on the left with the set of vectors in $W_{i}$. Instead of considering the cardinality of a set on the right hand side, we are considering the dimension of a vector space spanned by that set.

Rado's theorem has a slight generalization, which is a linear version of König's theorem. It will be the key input to our proof:

Proposition $3.2([9])$. If $V_{1}, \ldots, V_{r}$ are subspaces of an ambient space $V$, the maximum number of linearly independent representatives from different $V_{i}$ 's is

$$
\min _{I \subseteq[r]}\left[\operatorname{dim}\left(\sum_{i \in I} V_{i}\right)+r-|I|\right] .
$$

Moreover, suppose that $I \subset[r]$ is a subset attaining the minimum in the theorem. Then any system of linearly independent representatives must use a basis of $\sum_{i \in I} V_{i}$ as well as one vector from each of the spaces $V_{j}$ for $j \in[r] \backslash I$.

\section{Main TheOREM}

Let us now describe our main result. Let $\mathcal{O}$ be a discrete valued ring and $\mathcal{K}$ its field of fractions. Although all the arguments in this section work in this generality, for the purposes of the later application to higher laminations, we will take $\mathcal{O}=\mathbb{F}[[t]]$ and $\mathcal{K}=\mathbb{F}((t))$ for some field $\mathbb{F}$. The valuation $\operatorname{val}(x)$ of an element

$$
x=\sum_{i=-N}^{\infty} a_{i} t^{i} \in \mathcal{K}
$$

is the minimum $i$ such that $a_{i} \neq 0$.

We are interested in rank $n, \mathcal{O}$-submodules of $\mathcal{K}^{n}$. Such full-rank $\mathcal{O}$-submodules are called lattices. 
Let $L_{1}, L_{2}, \ldots, L_{n}$ be lattices in $\mathcal{K}^{n}$. We wish to find the maximum value of

$$
-\operatorname{val}\left(\operatorname{det}\left(v_{1}, \ldots, v_{n}\right)\right)
$$

under the condition that $v_{i} \in L_{i}$. Equivalently, we would like to find the minimum value of $\operatorname{val}\left(\operatorname{det}\left(v_{1}, \ldots, v_{n}\right)\right)$. Let us then define

$$
A\left(L_{1}, L_{2}, \ldots, L_{n}\right)=\min \left\{\operatorname{val}\left(\operatorname{det}\left(v_{1}, \ldots, v_{n}\right)\right) \mid v_{i} \in L_{i}\right\} .
$$

It is not difficult to see that the minimum value is attained for generic choices of $v_{1}, \ldots, v_{n}$ using the upper semi-continuity of the valuation function.

Given any vector $w \in \mathcal{K}^{n}$, let us define

$$
c\left(w, L_{i}\right):=\min \left\{\lambda \in \mathbb{Z} \mid t^{\lambda} w \in L_{i}\right\} .
$$

It will be convenient for us to define

$$
c\left(L, L_{i}\right)=\min \left\{c\left(w, L_{i}\right) \mid w \text { is a generator of } L\right\} .
$$

Here, $w \in L$ is a generator of $L$ if and only if $w$ belongs to some set of vectors in $\mathcal{K}^{n}$ which form a basis for $L$ as an $\mathcal{O}$-module. We will say that $w$ is a tight generator for $L$ with respect to $L_{i}$ if $w$ is a generator for $L$ and $c\left(w, L_{i}\right)=c\left(L, L_{i}\right)$. Let us remark that it is immediate from this definition that if $v_{i} \in L_{i}$, then $t^{-c\left(L, L_{i}\right)} v_{i} \in L$.

Example 4.1. Let $n=3$. Let $e_{1}, e_{2}, e_{3}$ be the standard basis of $\mathcal{K}^{3}$. Consider the standard lattice $L=\left\langle e_{1}, e_{2}, e_{3}\right\rangle$. Let $v=t^{3} e_{1}-3 t^{2} e_{2}$. Then $c(v, L)=-2$.

Now let $M$ be the lattice $\left\langle t^{2} e_{1}, t e_{2}, t^{-1} e_{3}\right\rangle$. Then $c(M, L)=-2$.

Let us define

$$
\operatorname{val}(\operatorname{det}(L))=A(L, L, \ldots, L) .
$$

(In other words, we take $L_{i}=L$ for all $i$ in the expression $A\left(L_{1}, L_{2}, \ldots, L_{n}\right)$.) The expression $\left\{\operatorname{val}\left(\operatorname{det}\left(v_{1}, \ldots, v_{n}\right)\right) \mid v_{i} \in L\right\}$ is minimized exactly when $v_{1}, \ldots, v_{n}$ form a set of generators for the lattice $L$.

Proposition 4.2. For any lattice $L$, we have

$$
\operatorname{val}(\operatorname{det}(L))+\sum_{i=1}^{n} c\left(L, L_{i}\right) \leqslant A\left(L_{1}, L_{2}, \ldots, L_{n}\right) .
$$

Let us give a quick proof. If

$$
A\left(L_{1}, L_{2}, \ldots, L_{n}\right)=\operatorname{val}\left(\operatorname{det}\left(v_{1}, \ldots, v_{n}\right)\right)
$$

where $v_{i} \in L_{i}$, then $t^{-c\left(L, L_{i}\right)} v_{i} \in L$, so that the vectors $t^{-c\left(L, L_{i}\right)} v_{i}$ generate some sublattice of $L$, and so

$\operatorname{val}(\operatorname{det}(L)) \leqslant \operatorname{val}\left(\operatorname{det}\left(t^{-c\left(L, L_{1}\right)} v_{1}, \ldots, t^{-c\left(L, L_{n}\right)} v_{n}\right)\right)=A\left(L_{1}, L_{2}, \ldots, L_{n}\right)-\sum_{i=1}^{n} c\left(L, L_{i}\right)$.

We use that if $L^{\prime}$ is a sublattice of $L$, then $\operatorname{val}(\operatorname{det}(L)) \leqslant \operatorname{val}\left(\operatorname{det}\left(L^{\prime}\right)\right)$.

The previous fairly simple proposition is complemented by the following theorem, which is the main theorem of this paper, and whose proof is more involved:

THEOREM 4.3. There exists a lattice $L$ for which we have the equality

$$
\operatorname{val}(\operatorname{det}(L))+\sum_{i=1}^{n} c\left(L, L_{i}\right)=A\left(L_{1}, L_{2}, \ldots, L_{n}\right) .
$$

In particular, this means:

(1) There exist $w_{i} \in L$ such that if we define $v_{i}:=t^{c\left(L, L_{i}\right)} w_{i}$ then $v_{i} \in L_{i}$. In other words, the $w_{i}$ are tight generators for $L$ with respect to $L_{i}$.

(2) The $w_{i}$ generate $L$. 
As we shall see, this theorem is a linearized version of the Kuhn-Munkres theorem, and in order to prove it, we generalize the Kuhn-Munkres algorithm to give an algorithm for finding such an $L$.

REMARK 4.4. The above theorem is a statement of max-min type. For any choice of $w_{i} \in L_{i}$, we have that

$$
A\left(L_{1}, L_{2}, \ldots, L_{n}\right) \leqslant \operatorname{val}\left(\operatorname{det}\left(w_{1}, \ldots, w_{n}\right)\right) .
$$

Thus for any choice of $w_{i} \in L_{i}$ and any choice of a lattice $L$, we have that

$$
\operatorname{val}(\operatorname{det}(L))+\sum_{i=1}^{n} c\left(L, L_{i}\right) \leqslant \operatorname{val}\left(\operatorname{det}\left(w_{1}, \ldots, w_{n}\right)\right)
$$

The above theorem says that the minimal value of the right hand side is the maximal value of the left hand side. We may therefore interpret the theorem as saying that we have found a lattice $L$ which is a witness to the minimal value of $\operatorname{val}\left(\operatorname{det}\left(w_{1}, \ldots, w_{n}\right)\right)$.

REMARK 4.5. Let $e_{1}, \ldots e_{n}$ be a basis of $\mathcal{K}^{n}$. Then we can recover the usual KuhnMunkres theorem by considering lattices of the form

$$
L_{i}=\left\langle t^{c_{i 1}} e_{1}, t^{c_{i 2}} e_{2}, \ldots, t^{c_{i n}} e_{n}\right\rangle .
$$

For more details, see [9].

Proof of Theorem 4.3. We will start with an arbitrary lattice $L$. At each step we will modify the lattice $L$ until we find one which satisfies the equality.

First note that $n$ vectors $w_{1}, \ldots, w_{n}$ will generate $L$ if and only if their images $\tilde{w}_{1}, \ldots, \tilde{w}_{n}$ in $L / t L$ form a basis.

For each $L_{i}$, let $W_{i} \subset L$ be the set of tight generators for $L$ with respect to $L_{i}$. Then let $\tilde{W}_{i} \subset L / t L$ be the projection of this set to $L / t L$. It is easy to verify that $\tilde{W}_{i}$ is a vector subspace of $L / t L$. We wish to choose one vector $\tilde{w}_{i}$ from each $\tilde{W}_{i}$ such that the $\tilde{w}_{1}, \ldots, \tilde{w}_{n}$ form a basis of $L / t L$. In other words, for the subspaces $\tilde{W}_{i}$, we wish to find a system of linearly independent representatives.

We are now equipped to explain the basic idea of the algorithm. Recall that for any $L$, we have

$$
\operatorname{val}(\operatorname{det}(L))+\sum_{i=1}^{n} c\left(L, L_{i}\right) \leqslant A\left(L_{1}, L_{2}, \ldots, L_{n}\right),
$$

where equality holds exactly when we have a system of linearly independent representatives of the subspaces $\tilde{W}_{i}$. At each stage, we will either make the left hand side bigger by some integer value, or we will increase the size of the system of linearly independent representatives. The only way this terminates is that we have a system of $n$ linearly independent representatives.

Here is the algorithm for finding $L$. Start with an arbitrary lattice $L$.

We have the subspace $\tilde{W}_{i} \subset L / t L$ for each $i \leqslant n$. Start by finding the maximum number of linearly independent representatives $\tilde{w}_{i}$ for $i \in J$, where $J \subset[n]$. Then we have that by Theorem 3.2, there exists a set $I \subset[n]$ such that

$$
|J|=\operatorname{dim}\left(\sum_{i \in I} \tilde{W}_{i}\right)+n-|I| .
$$

Moreover, we have that among the $\tilde{w}_{i}$, some form a basis for $\tilde{W}:=\sum_{i \in I} \tilde{W}_{i}$, while the rest have indices in $[n] \backslash I$. Thus we may write $J=J_{1} \amalg J_{2}$, where $\tilde{w}_{i}$ for $i \in J_{1}$ give a basis of $\tilde{W}$, and $J_{2} \subset[n] \backslash I$. 
Choose any lift of $\tilde{W}$ to an $\mathcal{O}$-submodule of $L$. Call this lift $W \subset L$. Now let $L^{\prime}=t^{-1} W+L$. We claim that

$$
\operatorname{val}(\operatorname{det}(L))+\sum_{i=1}^{n} c\left(L, L_{i}\right) \leqslant \operatorname{val}\left(\operatorname{det}\left(L^{\prime}\right)\right)+\sum_{i=1}^{n} c\left(L^{\prime}, L_{i}\right) .
$$

To see this, first note that

$$
\operatorname{val}\left(\operatorname{det}\left(L^{\prime}\right)\right)=\operatorname{val}(\operatorname{det}(L))-\operatorname{dim} W .
$$

Also note that

$$
\begin{aligned}
& c\left(L^{\prime}, L_{i}\right)=c\left(L, L_{i}\right)+1 \text { for } i \in I \\
& c\left(L^{\prime}, L_{i}\right)=c\left(L, L_{i}\right) \text { for } i \in[n] \backslash I .
\end{aligned}
$$

Note also that $\operatorname{dim} W \leqslant|I|$ with equality only when we have a complete system of linearly independent representatives. This yields the inequality (2).

This inequality tells us that, at each stage, if we have not found a complete system of linearly independent representatives, we may modify $L$ to get a lattice $L^{\prime}$ for which the inequality is strictly closer. We can then iterate this process-find another maximum set of linearly independent representatives; if they still do not span, we can modify the lattice again. The process cannot continue indefinitely, and it will end with a lattice for which we have $n$ linearly independent representatives, and this lattice will satisfy the equality of our theorem.

REMARK 4.6. The algorithm for finding the lattice $L$ gives a simple way of computing

$$
A\left(L_{1}, L_{2}, \ldots, L_{n}\right) \text {. }
$$

Compare it with the alternative: one may pick $n$ generators of each of the lattices $L_{i}$. Then one may consider all $n^{n}$ determinants formed by choosing one generator from each lattice and look for the one with minimal valuation. One can do slightly better than this by choosing the generators carefully, but in any case, the computation will be much more laborious than the algorithm given above. Compare with Remark 2.4.

REMARK 4.7. Here are some heuristics which may help give a sense of the difficulties in finding $L$. For any lattice $L$, there are always tight generators for $L$ with respect to any other lattice $M$. The difficulty is finding tight generators $w_{i}$ for $L$ with respect to each of $L_{1}, \ldots, L_{n}$ such that the $w_{i}$ will generate $L$. Typically, we can find tight generators $w_{i}$ which may be linearly independent in $\mathcal{K}^{n}$, but they will not necessarily generate all of $L$. (And if we take the lattice $L^{\prime}$ spanned by the $w_{i}$, the $w_{i}$ may not be tight generators for $L^{\prime}$.)

EXAMPLE 4.8. Consider the lattices

$$
\begin{aligned}
& L_{1}=\left\langle e_{1}, e_{2}, e_{3}\right\rangle \\
& L_{2}=\left\langle t^{3} e_{1}, e_{2}, t^{-3} e_{3}\right\rangle, \\
& L_{3}=\left\langle t^{4} e_{1},-\left(t+t^{2}\right) e_{1}-t^{-1} e_{2}, e_{1}+t^{-2} e_{2}+t^{-3} e_{3}\right\rangle .
\end{aligned}
$$

Let us apply our algorithm to find $L$ satisfying the theorem.

We can start with any $L$, for example $L=\left\langle e_{1}, e_{2}, e_{3}\right\rangle$. We have $\operatorname{val}(\operatorname{det}(L))=0$, $c\left(L, L_{1}\right)=0, c\left(L, L_{2}\right)=c\left(L, L_{3}\right)=-3$, while

$$
A\left(L_{1}, L_{2}, L_{3}\right)=\operatorname{val}\left(\operatorname{det}\left(e_{1}, t^{-3} e_{3}, e_{1}+t^{-2} e_{2}+t^{-3} e_{3}\right)\right)=-5 .
$$

The tight generators for $L$ with respect to $L_{1}$, when projected to $L / t L$ give the three-dimensional vector space $W_{1}$ spanned by $e_{1}, e_{2}, e_{3}$. The tight generators for $L$ with respect to $L_{2}$ or $L_{3}$, when projected to $L / t L$ give the one-dimensional vector 
space $W_{2}=W_{3}$ spanned by $e_{3}$. The maximum number of linearly independent representatives is 2 . Then let $I=\{2,3\}$, so that $W=W_{2}+W_{3}$ is the one-dimensional space spanned by $e_{3}$. Let $L^{\prime}=t^{-1} W+L=\left\langle e_{1}, e_{2}, t^{-1} e_{3}\right\rangle$.

It turns out that our algorithm terminates after one step, as $\operatorname{val}\left(\operatorname{det}\left(L^{\prime}\right)\right)=-1$, $c\left(L, L_{1}\right)=0, c\left(L, L_{2}\right)=c\left(L, L_{3}\right)=-2$.

The reader may also try starting with $L=\left\langle e_{1}, e_{2}, t e_{3}\right\rangle$. It will then take two steps to implement the algorithm.

\section{Buildings And LAMinations}

We can now apply our main theorem, Theorem 4.3, to the study of intersection pairings between higher laminations. These intersection pairings extend the intersection pairing between laminations on a surface defined by Thurston, which arise in the special case when $G=S L_{2}$. (See [2] for the relationship between laminations and cluster algebras in this case.)

The next few subsections will introduce higher laminations and translate the main theorem into this language. We start by introducing the objects used to define higher laminations, the affine Grassmannian and the affine building. We will then describe some invariants $f_{i j k}^{t}$ of configurations in the affine Grassmannian and the affine building that are tropicalizations of invariants of configurations of flags. Our main theorem, once translated to this context, will give an interpretation of the functions $f_{i j k}^{t}$ in terms of the metric geometry of the building.

5.1. Affine Grassmannian and Affine Buildings. Let $G$ be one of the groups $G L_{n}, P G L_{n}$ or $S L_{n}$. Write $G^{\vee}$ for its Langlands dual group, $G L_{n}, S L_{n}$ or $P G L_{n}$, respectively. Let $\mathbb{F}$ be a field, which for our purposes will always be $\mathbb{R}$ or $\mathbb{C}$. Let $\mathcal{O}=\mathbb{F}[[t]]$ and $\mathcal{K}=\mathbb{F}((t))$ as before. Note that $\mathcal{O}$ is naturally a valuation ring.

The affine Grassmannian for $G$ is an (ind-)scheme whose $F$-points are the set

$$
\operatorname{Gr}(\mathbb{F})=\operatorname{Gr}(G)=G(\mathcal{K}) / G(\mathcal{O}) .
$$

Here is a concrete description of this set when $G=G L_{n}, P G L_{n}$ and $S L_{n}$. For $G=G L_{n}$, a point in the affine Grassmannian is given by a lattice in $\mathcal{K}_{n}$ (a finitely generated, rank $n, \mathcal{O}$-submodule of $\left.\mathcal{K}^{n}\right)$. For $G=S L_{n}$, a point in the affine Grassmannian corresponds to a lattice which has the property that this lattice has generators $v_{1}, \ldots, v_{n}$ such that

$$
v_{1} \wedge \cdots \wedge v_{n}=e_{1} \wedge \cdots \wedge e_{n}
$$

Here $e_{1}, \ldots, e_{n}$ is the standard basis of $\mathcal{K}^{n}$. For $G=P G L_{n}$, a point in the affine Grassmannian corresponds to an equivalence class of lattices up to scale: two lattices $L$ and $L^{\prime}$ are equivalent if $L=c L^{\prime}$ for some $k \in \mathcal{K}$. In all three cases, the affine Grassmannian consists of some set of lattices. Moreover, in each case, $G(\mathcal{K})$ acts on the set of such lattices, and the stabilizer of any lattice is isomorphic to $G(\mathcal{O})$.

The affine Grassmannian has a metric naturally taking values in the dominant coweights of $G$. Recall that the coweight lattice $\Lambda$ is defined as $\operatorname{Hom}\left(\mathbf{G}_{m}, T\right)$ (the set of group homomorphisms from a one-dimensional torus into the maximal torus $T \subset G)$. The dominant coweights are those coweights lying in the dominant cone. For example, for $G=G L_{n}$, the set of dominant coweights is exactly the set of

$$
\mu=\left(\mu_{1}, \ldots, \mu_{n}\right) \in \mathbb{Z}^{n}
$$

where $\mu_{1} \geqslant \mu_{2} \geqslant \cdots \geqslant \mu_{n}$. For $G=S L_{n}$ and $P G L_{n}$, the coweights are given by similar conditions. For $S L_{n}$, the dominant coweights further satisfy the relation $\mu_{1}+$ $\cdots+\mu_{n}=0$. For $P G L_{n}$, the dominant coweights lie in the quotient $\mathbb{Z}^{n} /(1,1, \ldots, 1)$. 
Pairs of elements of $\mathrm{Gr}$ up to the action of $G(\mathcal{K})$ are in bijection with double cosets

$$
G(\mathcal{O}) \backslash G(\mathcal{K}) / G(\mathcal{O}) .
$$

These cosets are parameterized by the set $\Lambda_{+}$of dominant coweights of $G$. Let us explain further.

Fix a basis $e_{1}, \ldots, e_{n}$ of $\mathcal{K}^{n}$. For any dominant coweight $\mu=\left(\mu_{1}, \ldots, \mu_{m}\right)$, we can consider the element of $G(\mathcal{K})$ which is given by the matrix with diagonal entries $t^{-\mu_{i}}$. Applying this element to the trivial lattice $\left\langle e_{1}, \ldots, e_{n}\right\rangle$ gives us a lattice which we will call $t^{\mu}$. Any two points $p$ and $q$ of the affine Grassmannian can be translated by some element of $G(\mathcal{K})$ to $t^{0}$ and $t^{\mu}$, respectively. It turns out that $\mu$ will be unique. This gives the identification of the double coset space with $\Lambda_{+}$.

For $p$ and $q$ as above, we will write

$$
d(p, q)=\mu
$$

and say that the distance from $p$ to $q$ is $\mu$. Note that this is a non-symmetric distance function. We have the relation

$$
d(q, p)=-w_{0} d(p, q)
$$

where $w_{0}$ is the longest element of the Weyl group of $G$. There is a partial order on $\Lambda^{+}$ defined by $\lambda>\mu$ if $\lambda-\mu$ is a positive linear combination of positive co-roots). Under this partial ordering, the distance function satisfies a version of the triangle inequality. If we take this metric on the affine Grassmannian, then $G(\mathcal{K})$ acts by isometries.

We can now introduce the affine building for $G=P G L_{n}$, the case which is of the most interest to us. The affine building is a simplicial complex which captures the geometry of the above metric on the affine Grassmannian.

The set of vertices of the affine building for $P G L_{n}$ are in bijection with the points of the affine Grassmannian $\operatorname{Gr}\left(P G L_{n}\right)$. The simplices of the affine building are as follows: for any lattices $L_{0}, L_{1}, \ldots, L_{k}$, there is a $k$-simplex with vertices at $L_{0}, L_{1}, \ldots, L_{k}$ if and only if

$$
L_{0} \subset L_{0} \subset \cdots \subset L_{k} \subset t^{-1} L_{0} .
$$

(Recall that $\operatorname{Gr}\left(P G L_{n}\right)$ consists of lattices up to scale. For this reason, we will need to choose appropriate representatives $L_{0}, \ldots, L_{k}$. Note that it may be necessary to scale some of the $L_{i}$ in order to fulfill this condition.) The non-symmetric, coweight-valued metric we defined above descends to a metric on the affine building.

5.2. Invariants of Flags. We now define some functions of triples of principal affine flags as well as their tropical analogues, which are functions of triples of points in the affine Grassmannian.

We start by defining principal affine flags for $G=S L_{n}$. We will work over a field $\mathbb{F}$. The space of principal affine flags is parameterized by the quotient $G / U$, where $U \subset G$ is the subgroup of unipotent upper triangular matrices. Concretely, we can specify a principal affine flag by giving an ordered basis $v_{1}, \ldots, v_{n}$ for a vector space over $\mathbb{F}$. These $n$ vectors determine a flag, where the $k$-dimensional subspace of this flag is spanned by $v_{1}, \ldots, v_{k}$ for $k \leqslant n$. Additionally, the data of a principal affine flag includes the volume forms

$$
v_{1} \wedge \cdots \wedge v_{k}
$$

on these subspaces. Finally, we require that

$$
v_{1} \wedge \cdots \wedge v_{n}
$$

is the standard volume form (without this requirement, we would be dealing with $G L_{n}$ flags). Two sets of basis vectors will determine the same principal affine flag if 
they give the same $k$-forms $v_{1} \wedge \cdots \wedge v_{k}$ for $k \leqslant n$. We will sometimes abbreviate "principal affine flags" by calling them principal flags.

We would like to consider the space of three flags up to the left diagonal action of $G$ :

$$
G \backslash(G / U)^{3} .
$$

Let $F_{1}, F_{2}, F_{3}$ be three flags which are represented by bases $u_{1}, \ldots, u_{n}, v_{1}, \ldots, v_{n}$ and $w_{1}, \ldots, w_{n}$ respectively. For non-negative integers $i, j, k$ such that $i+j+k=n$ we can consider the function

$$
f_{i j k}\left(F_{1}, F_{2}, F_{3}\right)=\operatorname{det}\left(u_{1}, u_{2}, \ldots, u_{i}, v_{1}, v_{2}, \ldots v_{j}, w_{1}, w_{2}, \ldots, w_{k}\right) .
$$

This function is manifestly $G$-invariant. Note that if one of $i, j, k$ is 0 , these functions only depend on two of the flags. We call such functions edge functions, while the functions depending on three flags will be called face functions.

REMARK 5.1. The functions $f_{i j k}$ were used to define the cluster structure on configuration spaces of flags in [1]. These are building blocks for the cluster structure on the space $\mathcal{A}_{G, S}$. The space of configurations of $m$ principal flags is $\mathcal{A}_{G, S}$ where $S$ is a disc with $m$ marked points. For any triangulation of an $m$-gon, the edge and face functions form a cluster for the space of configurations of $m$ principal flags.

The functions $f_{i j k}$ have tropical analogues, $f_{i j k}^{t}$, which will be functions of three points in the affine Grassmannian for $S L_{n}$. The functions $f_{i j k}^{t}$ were first defined in [6], where they were called $H_{i j k}$.

Let $x_{1}, x_{2}, x_{3}$ be three points in the affine Grassmannian for $S L_{n}$, thought of as lattices. For $i, j, k$ as above with $i+j+k=n$, we consider

$$
-\operatorname{val}\left(\operatorname{det}\left(u_{1}, \ldots, u_{i}, v_{1}, \ldots v_{j}, w_{1}, \ldots, w_{k}\right)\right)
$$

as $u_{1}, \ldots, u_{i}$ range over elements of the $\mathcal{O}$-submodule $x_{1}, v_{1}, \ldots, v_{j}$ range over elements of $x_{2}$, and $w_{1}, \ldots, w_{k}$ range over elements of $x_{3}$. Define $f_{i j k}^{t}\left(x_{1}, x_{2}, x_{3}\right)$ as the maximum value attained by this quantity.

REMARK 5.2. The edge functions recover the distance between two points in the affine Grassmannian. More precisely, for $i+j=n$, let us define

$$
f_{i j}^{t}\left(x_{1} x_{2}\right):=f_{i j 0}^{t}\left(x_{1}, x_{2}, x_{3}\right) .
$$

Then we have

$$
f_{i j}^{t}\left(x_{1} x_{2}\right)=\omega_{j} \cdot d\left(x_{1}, x_{2}\right)=\omega_{i} \cdot d\left(x_{2}, x_{1}\right) .
$$

Here $\omega_{i}$ and $\omega_{j}$ are fundamental weights for $S L_{n}$.

The functions $f_{i j k}^{t}$ have a straightforward extension to any triple of $G L_{n}$ lattices. Let us call that extension $\tilde{f}_{i j k}^{t}$. This allows us to extend $f_{i j k}^{t}$ to a function on the affine Grassmannian for $P G L_{n}$ in the following way. Let $x_{1}, x_{2}, x_{3}$ be three points in the affine Grassmannian for $P G L_{n}$, represented by three lattices $L_{1}, L_{2}, L_{3}$. For $i, j, k$ as above, we can again maximize

$$
-\operatorname{val}\left(\operatorname{det}\left(u_{1}, \ldots, u_{i}, v_{1}, \ldots v_{j}, w_{1}, \ldots, w_{k}\right)\right)
$$

as before. Call the resulting maximum

$$
\tilde{f}_{i j k}^{t}\left(L_{1}, L_{2}, L_{3}\right) \text {. }
$$

Note that $\tilde{f}_{i j k}^{t}\left(L_{1}, L_{2}, L_{3}\right)$ will depend on the representative lattices $L_{1}, L_{2}, L_{3}$ that we chose, which are only determined up to scale. To fix this, we renormalize, defining (3)

$$
f_{i j k}^{t}\left(x_{1}, x_{2}, x_{3}\right):=\tilde{f}_{i j k}^{t}\left(L_{1}, L_{2}, L_{3}\right)+\frac{i \operatorname{val}\left(\operatorname{det}\left(L_{1}\right)\right)+j \operatorname{val}\left(\operatorname{det}\left(L_{2}\right)\right)+k \operatorname{val}\left(\operatorname{det}\left(L_{3}\right)\right)}{n} .
$$


Note that if $L_{1}, L_{2}, L_{3}$ have determinant 1 (and hence correspond to points in the affine Grassmannian for $S L_{n}$ ) our definition reduces to the previous definition.

REMARK 5.3. The functions $f_{i j k}^{t}$ and $d_{i}$ are related to those used in our main theorem, Theorem 4.3. Observe that

- $\tilde{f}_{n}^{t}(L)=-A(L, L, \ldots, L)=-\operatorname{val}(\operatorname{det}(L))$, so that we always have $f_{n}^{t}(L)=0$,

- $\tilde{f}_{i j k}^{t}\left(L_{1}, L_{2}, L_{3}\right)=-A(\underbrace{L_{1}, \ldots, L_{1}}_{i}, \underbrace{L_{2}, \ldots, L_{2}}_{j}, \underbrace{L_{3}, \ldots, L_{3}}_{k})$,

- $d_{1}\left(L, L^{\prime}\right)=-c\left(L, L^{\prime}\right)-\frac{1}{n} \tilde{f}_{n}\left(L^{\prime}\right)+\frac{1}{n} \tilde{f}_{n}(L)=\tilde{f}_{n-1,1}\left(L, L^{\prime}\right)+\frac{(n-1) \operatorname{val}(\operatorname{det}(L))}{n}+$ $\frac{\operatorname{val}\left(\operatorname{det}\left(L^{\prime}\right)\right)}{n}$,

- $-c\left(L, L^{\prime}\right)=\tilde{f}_{n-1,1}\left(L, L^{\prime}\right)+\operatorname{val}(\operatorname{det}(L))$.

The functions of the type $f_{i j k}^{t}$ or $d_{i}$ therefore differ from functions of the type $A$ or $c$ by normalizations and a change of sign. We chose to use the functions $A$ and $c$ in the first part of this paper because they were better suited to the arguments of Theorem 4.3. However, the functions $f_{i j k}^{t}$ or $d_{i}$ are in some ways more fundamental, and better suited to the study of buildings and laminations.

It is straightforward to define similar functions

$$
f_{i_{1} i_{2} \ldots i_{k}}^{t}\left(x_{1}, x_{2} \ldots, x_{k}\right)
$$

where $i_{1}+i_{2}+\cdots+i_{k}=n$. Here, $x_{1}, x_{2}, \ldots, x_{k}$ are points in the affine building for $P G L_{n}$ or $S L_{n}$.

5.3. Metric interpretation of the Functions $f_{i j k}^{t}$. We now give another way to compute the tropical functions $f_{i j k}^{t}$. Whereas $f_{i j k}^{t}$ was defined in a valuation-theoretic way, it turns out that it is determined by the coweight-valued metric on the affine Grassmannian (and affine building).

We need some notation first. Let $\omega_{i}$ be the $i$-th fundamental weight for $S L_{n}$ : $\omega_{i}=(1, \ldots, 1,0, \ldots, 0)$ where there are $i 1$ 's and $n-i$ 's. Recall that for any two points $p, q$ in the affine Grassmannian, $d(p, q)$ is an element of the coweight lattice for $S L_{n}$.

Define, for $1 \leqslant i \leqslant n-1$,

$$
d_{i}(L, M)=\omega_{i} \cdot d(L, M) .
$$

Note that if $L, M$ are both in the affine Grassmannian for $S L_{n}$, then $d_{i}(L, M)$ is an integer for all $i$, while if they are in the affine Grassmannian for $P G L_{n}, d_{i}(L, M)$ may have denominator $n$.

We will extend the definition of $d_{i}(L, M)$ to the case when $L, M$ are both in the affine Grassmannian for $P G L_{n}$. We will need to view the coweight lattice of $P G L_{n}$ as containing the coweight lattice of $S L_{n}$ as an index $n$ sublattice. Recall that the coweight lattice for $S L_{n}$ is the subset of $\mathbb{Z}^{n}$ given by

$$
\left\{\left(x_{1}, \ldots, x_{n}\right) \mid x_{1}+\cdots+x_{n}=0\right\} .
$$

The coweight lattice for $P G L_{n}$ is given by

$$
\mathbb{Z}^{n} /(1,1, \ldots, 1)
$$

Any point in $\mathbb{Z}^{n}$ can be translated into the plane $x_{1}+\cdots+x_{n}=0$ using some multiple of $(1,1, \ldots, 1)$, at the cost of possibly introducing entries in $\frac{1}{n} \mathbb{Z}$. One can then easily see that the weight lattice for $P G L_{n}$ naturally contains as an index $n$ sublattice the coweight lattice of $S L_{n}$. 
Therefore if $p$ and $x$ are in the affine Grassmannian for $P G L_{n}$, viewing the coweights of $P G L_{n}$ in the same space as the coweights of $S L_{n}$, we can define pairings with $S L_{n}$ weights. Thus we may consider the quantities

$$
d_{i}(p, x):=\omega_{i} \cdot d(p, x) .
$$

These quantities lie in $\frac{1}{n} \mathbb{Z}$. Finally, note that

$$
d_{i}(p, x)=d_{n-i}(x, p) .
$$

Here is our main theorem giving a metric interpretation of the functions $f_{i j k}^{t}$. The most general form can be found in Theorem 5.10.

THEOREM 5.4. Let $x_{1}, x_{2}, x_{3}$ be any configuration of points in the affine Grassmannian for $P G L_{n}$. Then

$$
f_{i j k}^{t}\left(x_{1}, x_{2}, x_{3}\right)=\min _{p}\left[d_{i}\left(p, x_{1}\right)+d_{j}\left(p, x_{2}\right)+d_{k}\left(p, x_{3}\right)\right],
$$

where the minimum is taken over all $p$ in the affine Grassmannian for $P G L_{n}$.

REMARK 5.5. Note that both sides of the expression are in $\frac{1}{n} \mathbb{Z}$. A special case of the theorem is when $x_{1}, x_{2}, x_{3}$ is a configuration of points in the affine Grassmannian for $S L_{n}$. In this case, both sides of the expression are integers. The integrality on the left comes from a valuation, while the integrality on the right is slightly more subtle. In general, it is easy to check that the expression

$$
d_{i}\left(p, x_{1}\right)+d_{j}\left(p, x_{2}\right)+d_{k}\left(p, x_{3}\right)
$$

is determined mod 1 by $x_{1}, x_{2}, x_{3}$, i.e. it does not depend on $p$ when considered mod 1 . Clearly if $x_{1}, x_{2}, x_{3}, p$ are all in the affine Grassmannian for $S L_{n}$, the pairing between coweights and weights guarantees that the expression is integral, hence it is integral for any $p$.

REMARK 5.6. Note that even in the case that $x_{1}, x_{2}, x_{3}$ are all in the affine Grassmannian for $S L_{n}$, the minimizing point $p$ may be in the affine Grassmannian for $P G L_{n}$. For example, let $x_{1}$ correspond to the lattice $\left\langle e_{1}, e_{2}, e_{3}\right\rangle, x_{2}$ the lattice $\left\langle t e_{1}, t^{-1} e_{2}, e_{3}\right\rangle$, and $x_{3}$ the lattice $\left\langle t e_{1}, e_{2}, t^{-1} e_{3}\right\rangle$. There is a unique minimizing point $p$ given the lattice $\left\langle t e_{1}, e_{2}, e_{3}\right\rangle$ which is in the affine Grassmannian for $P G L_{n}$ but not for $S L_{n}$.

REMARK 5.7. We now have a description of the functions $f_{i j k}^{t}$ that is completely of a metric nature. Therefore the functions $f_{i j k}^{t}$ may also be naturally viewed as functions on configurations of points in the affine building. We observed in [7] that for positive configurations of points in the affine building, the functions $f_{i j k}^{t}$ only depended on metric properties of the configuration within the building. Moreover, we did not give an explicit formula for this dependence. We now have an explicit formula that holds for all configurations, not just positive ones.

Proof of Theorem 5.4. Let us now show how the theorems above follow from Theorem 4.3. We will give the proof in the case that $G=P G L_{n}$, which is more general than the case when $G=S L_{n}$.

We wish to show that

$$
f_{i j k}^{t}\left(x_{1}, x_{2}, x_{3}\right)=\min _{p} d_{i}\left(p, x_{1}\right)+d_{j}\left(p, x_{2}\right)+d_{k}\left(p, x_{3}\right) .
$$

First let $x_{1}, x_{2}, x_{3}$ be represented by lattices $L_{1}, L_{2}, L_{3}$. Let us take in the Theorem 4.3 the $n$ lattices

$$
\underbrace{L_{1}, \ldots, L_{1}}_{i}, \underbrace{L_{2}, \ldots, L_{2}}_{j}, \underbrace{L_{3}, \ldots, L_{3}}_{k}
$$


The theorem gives us that there exists a lattice $L$ such that

$$
\begin{aligned}
\operatorname{val}(\operatorname{det}(L))+i \cdot c\left(L, L_{1}\right)+j \cdot c\left(L, L_{1}\right) & +k \cdot c\left(L, L_{3}\right) \\
& =A\left(L_{1}, \ldots, L_{1}, L_{2}, \ldots, L_{2}, L_{3}, \ldots, L_{3}\right),
\end{aligned}
$$

where $A\left(L_{1}, \ldots, L_{1}, L_{2}, \ldots, L_{2}, L_{3}, \ldots, L_{3}\right)$ is the minimal value of

$$
\operatorname{val}\left(\operatorname{det}\left(u_{1}, \ldots, u_{i}, v_{1}, \ldots v_{j}, w_{1}, \ldots, w_{k}\right)\right)
$$

as $u_{1}, \ldots, u_{i}$ range over elements $L_{1}, v_{1}, \ldots v_{j}$ range over elements of $L_{2}$, and $w_{1}, \ldots, w_{k}$ range over elements of $L_{3}$.

Because

$$
A\left(L_{1}, \ldots, L_{1}, L_{2}, \ldots, L_{2}, L_{3}, \ldots, L_{3}\right)=-\tilde{f}_{i j k}^{t}\left(L_{1}, L_{2}, L_{3}\right),
$$

the theorem tells us that there are vectors

$$
u_{1}, \ldots, u_{i}, v_{1}, \ldots v_{j}, w_{1}, \ldots, w_{k}
$$

minimizing

$$
\operatorname{val}\left(\operatorname{det}\left(u_{1}, \ldots, u_{i}, v_{1}, \ldots v_{j}, w_{1}, \ldots, w_{k}\right)\right)
$$

such that the vectors

$$
\begin{gathered}
t^{-c\left(L, L_{1}\right)} u_{1}, \ldots, t^{-c\left(L, L_{1}\right)} u_{i} \\
t^{-c\left(L, L_{2}\right)} v_{1}, \ldots, t^{-c\left(L, L_{2}\right)} v_{j} \\
t^{-c\left(L, L_{3}\right)} w_{1}, \ldots, t^{-c\left(L, L_{3}\right)} w_{j}
\end{gathered}
$$

all lie in $L$, and moreover are a set of generators for $L$. Moreover, these vectors are tight generators for $L$ with respect to $L_{1}, L_{2}$, and $L_{3}$.

Let us unravel what this tells us. This means, for example, that if we view $d\left(L, L_{1}\right)$ as an element of $\mathbb{Z}^{n}$ (viewing $L$, and $L_{i}$ as $G L_{n}$ lattices temporarily), then $d\left(L, L_{1}\right)$ has as its first $i$ entries $-c\left(L, L_{1}\right)$. A simple calculation then tells us that

$$
d_{i}\left(L, L_{1}\right)=-i \cdot c\left(L, L_{1}\right)+\frac{i}{n}\left(\operatorname{val}\left(\operatorname{det}\left(L_{1}\right)\right)-\operatorname{val}(\operatorname{det}(L))\right) .
$$

Similarly,

$$
\begin{aligned}
& d_{j}\left(L, L_{2}\right)=-j \cdot c\left(L, L_{2}\right)+\frac{j}{n}\left(\operatorname{val}\left(\operatorname{det}\left(L_{2}\right)\right)-\operatorname{val}(\operatorname{det}(L))\right) . \\
& d_{k}\left(L, L_{3}\right)=-k \cdot c\left(L, L_{3}\right)+\frac{k}{n}\left(\operatorname{val}\left(\operatorname{det}\left(L_{3}\right)\right)-\operatorname{val}(\operatorname{det}(L))\right) .
\end{aligned}
$$

Putting this together with Equation (3) yields the result.

REMARK 5.8. The above theorem gives a way of computing $f_{i j k}^{t}\left(L_{1}, L_{2}, L_{3}\right)$, an invariant of three points in the affine building, in terms of distances between points in the building. The latter are easy to compute using Birkhoff factorization. In practice, the most efficient way to compute $f_{i j k}^{t}\left(L_{1}, L_{2}, L_{3}\right)$ is to find a lattice $L$ which is generated the appropriate tight vectors, as in the proof of the theorem. The proof therefore gives an algorithm for calculating $f_{i j k}^{t}\left(L_{1}, L_{2}, L_{3}\right)$. The only other method the author knows of is to find a basis of generators of $L_{1}, L_{2}, L_{3}$ and compute all the possible

$$
\left(\begin{array}{l}
n \\
i
\end{array}\right)\left(\begin{array}{l}
n \\
j
\end{array}\right)\left(\begin{array}{l}
n \\
k
\end{array}\right)
$$

determinants of various subsets of generators. (This is essentially how these functions were defined in [6].) Compare with Remarks 2.4, 4.6. 
EXAMPLE 5.9. The proof above shows that a lattice $L$ satisfying Equation (5) automatically satisfies Equation (4). However, the reverse is not true. In fact, this already fails for the functions $f_{i j}^{t}$ of two arguments. To take a simple example, let $L_{1}=\left\langle e_{1}, e_{2}, e_{3}\right\rangle$ and $L_{2}=\left\langle t^{-2} e_{1}, t^{-1} e_{2}, e_{3}\right\rangle$. Suppose we are interested in $f_{1,2}^{t}\left(L_{1}, L_{2}\right)$. If $L=\left\langle e_{1}, e_{2}, e_{3}\right\rangle$, we clearly have

$$
d_{1}\left(L, L_{1}\right)+d_{2}\left(L, L_{2}\right)=0+1=f_{1,2}^{t}\left(L_{1}, L_{2}\right)
$$

so that $L$ satisfies Equation (4). However

$$
\operatorname{val}\left(\operatorname{det}(L)+c\left(L, L_{1}\right)+2 c\left(L, L_{2}\right)=0+0+2 \cdot(-2)=-4\left\langle-3=A\left(L_{1}, L_{2}, L_{2}\right),\right.\right.
$$

so that $L$ does not satisfy Equation (5).

However we may take $L=\left\langle t^{-1} e_{1}, e_{2}, e_{3}\right\rangle$ so that

$$
d_{1}\left(L, L_{1}\right)+d_{2}\left(L, L_{2}\right)=\frac{1}{3}+\frac{2}{3}=f_{1,2}^{t}\left(L_{1}, L_{2}\right)
$$

and

$$
\operatorname{val}\left(\operatorname{det}(L)+c\left(L, L_{1}\right)+2 c\left(L, L_{2}\right)=-1+0+2 \cdot(-1)=-3=A\left(L_{1}, L_{2}, L_{2}\right) .\right.
$$

It is not hard to see that the theorem has the following mild generalization. Let $i_{1}+i_{2}+\cdots+i_{k}=n$. For points $x_{1}, x_{2}, \ldots, x_{k}$ in the affine building for $P G L_{n}$ or $S L_{n}$, we have that

\section{THEOREM 5.10.}

$$
f_{i_{1} i_{2} \ldots i_{k}}^{t}\left(x_{1}, x_{2} \ldots, x_{k}\right)=\min _{p}\left[d_{i_{1}}\left(p, x_{1}\right)+d_{i_{2}}\left(p, x_{2}\right)+\cdots+d_{i_{k}}\left(p, x_{k}\right)\right]
$$

where the minimum is taken over all $p$ in the affine Grassmannian for $P G L_{n}$.

REMARK 5.11. Note that if we phrase this theorem in terms of the functions purely in terms of lattices, it becomes somewhat more cumbersome. For example, we could write

$$
\tilde{f}_{i_{1} i_{2} \ldots i_{k}}^{t}\left(L_{1}, L_{2} \ldots, L_{k}\right)=\min _{L}\left[(-n+1) \tilde{f}_{n}^{t}(L)+\sum_{j=1}^{k} \tilde{f}_{n-i_{j}, i_{j}}^{t}\left(L, L_{j}\right)\right]
$$

where the minimum is taken over all lattices $L$ in the affine Grassmannian for $P G L_{n}$.

REMARK 5.12. Taking $i_{1}=i_{2}=\cdots=i_{n}=1$ in the above theorem, and then adjusting for normalizations, recovers Theorem 4.3.

5.4. Higher Laminations. We will now specialize to the case of positive configurations of points in the affine building, which give tropical points of $\mathcal{A}_{G, S}$ in the case that $S$ is a disc with marked points. First we recall the definition of a positive configurations in the affine building, which is a particular type of higher lamination.

DEFinition 5.13. Take $m$ points of the real affine Grassmannian,

$$
x_{1}, x_{2}, \ldots, x_{m}
$$

This configuration of points will be called a positive configuration of points in the affine Grassmannian if and only if we have a collection of ordered bases for each $x_{i}$,

$$
v_{i 1}, v_{i 2}, \ldots, v_{i n}
$$

such that for each triple of integers $p, q, r, 1 \leqslant p<q<r \leqslant m$, and each triple of non-negative integers $i, j, k$ such that $i+j+k=n$,

- $f_{i j k}^{t}\left(x_{p}, x_{q}, x_{r}\right)=-\operatorname{val}\left(\operatorname{det}\left(v_{p 1}, \ldots, v_{p i}, v_{q 1}, \ldots v_{q j}, v_{r 1}, \ldots, v_{r k}\right)\right)$, and more-
over

- the leading coefficient of $\operatorname{det}\left(v_{p 1}, \ldots, v_{p i}, v_{q 1}, \ldots v_{q j}, v_{r 1}, \ldots, v_{r k}\right)$ is positive. 
We can use the same definition when $G=S L_{n}$ or $P G L_{n}$.

REMARK 5.14. It is sufficient to check the above two conditions for only those triples $p, q, r$ that are vertices of a triangle in a particular triangulation of the $m$-gon. If the conditions hold in one triangulation, they hold in any other triangulation.

Positive configurations of points in the affine Grassmannian, up to metric equivalence given by the metric on the affine building, give the integral tropical points of $\mathcal{A}_{G, S}$, which we write $\mathcal{A}_{G, S}\left(\mathbb{Z}^{t}\right)$. More generally, the tropical points $\mathcal{A}_{G, S}\left(\mathbb{R}^{t}\right)$ are higher laminations.

5.5. Relationship to the Duality Conjectures. Cluster algebras naturally occur as rings of functions on cluster varieties. For any cluster variety $\mathcal{A}$, there is an associated cluster variety $\mathcal{X}$ as well as a map, $\pi: \mathcal{A} \rightarrow \mathcal{X}$, which intertwines cluster structures on both these varieties. The pair $(\mathcal{A}, \mathcal{X})$ forms what is called a cluster ensemble.

The duality conjectures of Fock and Goncharov relate the two spaces $\mathcal{A}_{G, S}$ and $\mathcal{X}_{G^{\vee}, S}$, where $G^{\vee}$ is the Langlands dual group of $G$. These two spaces are both cluster varieties, and they are conjectured to be mirror duals to each other.

The Fock-Goncharov conjectures roughly state that the tropical points of one space parameterize a canonical basis of functions on the other space. This means that, for example, $\mathcal{X}_{G^{\vee}, S}\left(\mathbb{Z}^{t}\right)$ parameterizes a basis of functions for $\mathcal{A}_{G, S}$. This bijection satisfies many compatibility relations which we will not discuss here. These conjectures are an active area of research, and have been proven in many cases by Goncharov and Shen [4], building on the work of Gross, Hacking, Keel and Kontsevich [5].

The work of [5] places these duality conjectures in the framework of mirror symmetry. They construct a canonical basis that they call the theta basis. The theta basis comes from the combinatorics of scattering diagrams, which in turn encode certain tropical curve counts on these spaces.

The duality conjectures further imply that there should be a pairing between tropical spaces:

$$
\mathcal{X}_{G^{\vee}, S}\left(\mathbb{Z}^{t}\right) \times \mathcal{A}_{G, S}\left(\mathbb{Z}^{t}\right) \rightarrow \mathbb{Z}
$$

This pairing will carry much combinatorial information about the cluster varieties $\mathcal{X}_{G^{\vee}, S}$ and $\mathcal{A}_{G, S}$.

Our goal here is to give a way to compute the intersection pairing which is explicit, and doesn't depend on construction of, for example, theta bases. Our computation foregrounds the tropical, piecewise-linear nature of the intersection pairing, and is geometric and combinatorial, whereas the constructions of [5] are recursive and fairly complicated.

Let us now explain how to construct this pairing. A tropical point $l \in \mathcal{X}_{G^{\vee}, S}\left(\mathbb{Z}^{t}\right)$ corresponds to a function $f_{l}$ on $\mathcal{A}_{G, S}$ by duality conjectures. A point $l^{\prime} \in \mathcal{A}_{G, S}\left(\mathbb{Z}^{t}\right)$ arises by taking valuations of some (positive) Laurent-series valued point $x_{l^{\prime}} \in$ $\mathcal{A}_{G, S}(\mathcal{K})$ :

Then we get a pairing $\mathcal{I}$ :

$$
-\operatorname{val}\left(x_{l^{\prime}}\right)=l^{\prime}
$$

$$
\mathcal{I}\left(l, l^{\prime}\right)=-\operatorname{val} f_{l}\left(x_{l^{\prime}}\right) .
$$

The value of $\mathcal{I}\left(l, l^{\prime}\right)$ is independent of the choice of the point $x_{l^{\prime}}$, because $f_{l}$ should be a Laurent polynomial in the cluster coordinates.

Alternatively, we can perform the dual construction. A lamination $l^{\prime} \in \mathcal{A}_{G, S}\left(\mathbb{Z}^{t}\right)$ corresponds to a function $f_{l^{\prime}}$ on $\mathcal{X}_{G^{\vee}, S}$. Then

$$
\mathcal{I}\left(l, l^{\prime}\right)=-\operatorname{val} f_{l^{\prime}}\left(x_{l}\right)
$$


where $-\operatorname{val}\left(x_{l}\right)=l$ for $x_{l} \in \mathcal{X}_{v G, S}(\mathcal{K})$. Surprisingly, these dual constructions conjecturally give the same answer.

The pairing $\mathcal{I}$ can be interpreted as an intersection pairing between higher laminations, once one identifies the tropical spaces $\mathcal{X}_{G^{\vee}, S}\left(\mathbb{Z}^{t}\right)$ and $\mathcal{A}_{G, S}\left(\mathbb{Z}^{t}\right)$ with higher laminations for the groups $G^{\vee}$ and $G$, respectively. When $G=S L_{2}, \mathcal{I}$ specializes to the usual intersection pairing between $\mathcal{A}$ - and $\mathcal{X}$-laminations on a surface $S([1])$.

The functions $f_{i j k}$ are particular examples of cluster variables on $\mathcal{A}_{G, S}$. Cluster variables on $\mathcal{A}_{G, S}$-in fact, all cluster monomials-are conjecturally part of the canonical basis parameterized by $\mathcal{X}_{G^{\vee}, S}\left(\mathbb{Z}^{t}\right)$. Consider a cluster for the space $\mathcal{A}_{G, S}$ consisting of functions $a_{1}, \ldots, a_{N}$. Let the corresponding cluster $\mathcal{X}$-variables on the space $\mathcal{X}_{G^{\vee}, S}$ be $x_{1}, \ldots, x_{N}$. (There is a notion of "corresponding variable" because $\mathcal{A}_{G, S}$ and $\mathcal{X}_{G^{\vee}, S}$ are part of dual cluster ensembles.) Then for integers $d_{i} \geqslant 0$ the cluster monomial

$$
a_{1}^{d_{1}} \cdots a_{N}^{d_{N}}
$$

should correspond to the tropical point in $\left(d_{1}, \ldots, d_{N}\right) \in \mathcal{X}_{G^{\vee}, S}\left(\mathbb{Z}^{t}\right)$ in the coordinate chart $x_{1}, \ldots, x_{N}$.

Theorem 4.3 gives a way of computing $f_{i j k}^{t}\left(l^{\prime}\right)$ for any point $l^{\prime} \in \mathcal{A}_{G, S}\left(\mathbb{Z}^{t}\right)$. Thus it gives a geometric interpretation of the intersection pairings.

Furthermore, for any cluster chart coming from a triangulation of $S$, the associated cluster monomials are parameterized by a cone in $\mathcal{X}_{G^{\vee}, S}\left(\mathbb{Z}^{t}\right)$. The tropical pairing is additive on this cone. In other words, if $f$ and $g$ are cluster monomials for the same cluster, we have

$$
(f g)^{t}(l)=f^{t}(l)+g^{t}(l) .
$$

Thus, for any cluster that consists of only functions of the type $f_{i j k}$ (there are many of these, one for each ideal triangulation of $S$ ), we can calculate $f^{t}$ whenever $f$ is a cluster monomial in such a cluster. We therefore understand the pairing between a union of cones in $\mathcal{X}_{G^{\vee}, S}\left(\mathbb{Z}^{t}\right)$ and the whole space $\mathcal{A}_{G, S}\left(\mathbb{Z}^{t}\right)$.

One would like to have a geometric interpretation of these intersection pairings in general, for all points of $\mathcal{X}_{G^{\vee}, S}\left(\mathbb{Z}^{t}\right)$ and $\mathcal{A}_{G, S}\left(\mathbb{Z}^{t}\right)$. We will discuss further steps towards this in Section 6.

REMARK 5.15. We close this section by mentioning a surprising consequence. First recall that the functions $f_{i j k l}$ satisfy various identities, for example, for $i+j+k+l=n$, we have:

$$
f_{i j k l} f_{i+1, j-1, k+1, l-1}=f_{i, j, k+1, l-1} f_{i+1, j-1, k, l}+f_{i+1, j, k, l-1} f_{i, j-1, k+1, l} .
$$

Then we can tropicalize this to get that evaluating the three functions

$$
\begin{gathered}
f_{i j k l}^{t}+f_{i+1, j-1, k+1, l-1}^{t}, \\
f_{i, j, k+1, l-1}^{t}+f_{i+1, j-1, k, l}^{t}, \\
f_{i+1, j, k, l-1}^{t}+f_{i, j-1, k+1, l}^{t}
\end{gathered}
$$

on four points in the affine building gives three numbers such that the two largest of these numbers are equal. However, if we use the metric interpretation of the functions $f_{i j k l}^{t}$, this statement seems to be fairly non-trivial.

\section{Generalizations}

We explained in the previous section how our metric formula for the function $f_{i j k}^{t}$ gave a way to compute intersection pairings between a subset of $\mathcal{X}_{G^{\vee}, S}\left(\mathbb{Z}^{t}\right)$ and $\mathcal{A}_{G, S}\left(\mathbb{Z}^{t}\right)$. The key observation was that if $f$ is a cluster variable in some cluster, then giving a metric interpretation of $f^{t}$ gives us a way of computing some set of intersection pairings. More precisely, if $f_{l}$ is the function on $\mathcal{A}_{G, S}\left(\mathbb{Z}^{t}\right)$ which corresponds to the 
tropical point $l \in \mathcal{X}_{G^{\vee}, S}\left(\mathbb{Z}^{t}\right)$, then giving a metric interpretation of $f_{l}^{t}$ is the same as computing $\mathcal{I}(l,-)$. Our goal for this section will therefore be to give a metric formula for $f^{t}$ for several more instances where $f$ is a cluster variable.

One can show using the sequence of mutations for a flip and an inductive argument that the functions

$$
f_{i_{1} i_{2} \ldots i_{k}}
$$

are cluster variables. Then Theorem 5.10 gives a metric formula for

$$
f_{i_{1} i_{2} \ldots i_{k}}^{t}
$$

We will now show how this can be further extended to other cluster variables.

6.1. Tropicalization of Functions. We begin by recalling from [3] and [7] how to evaluate $f^{t}$ on a higher lamination for a cluster variable $f$. Our treatment will be slightly different from those papers, and will be tailored to our particular goals.

Let $\mathcal{A}$ denote the variety of principal affine flags, $G / U$. The space of configurations of $m$ flags, denoted $\operatorname{Conf}_{m}(\mathcal{A})$, the quotient of $(G / U)^{n}$ by the diagonal action of $G$.

It is well-known that the functions on $\mathcal{A}_{G}$ are naturally isomorphic to

$$
\bigoplus_{\lambda \in \Lambda_{+}} V_{\lambda}
$$

as a $G$-representation under the left action of $G$. Moreover, it is a fact that any cluster variable $f$ in the space of functions $\mathcal{O}\left(\operatorname{Conf}_{m}(\mathcal{A})\right)$ is given by an invariant in

$$
\left[V_{\lambda_{1}}^{*} \otimes V_{\lambda_{2}}^{*} \otimes \cdots \otimes V_{\lambda_{m}}^{*}\right]^{G}
$$

for some set of dominant weights $\lambda_{i}$. Here, $V^{*}$ is the representation dual to $V$. We use the dual representations here for convenience.

For example, the functions $f_{i j k}$ defined previously are given by invariants in

$$
\left[V_{\omega_{i}}^{*} \otimes V_{\omega_{j}}^{*} \otimes V_{\omega_{k}}^{*}\right]^{G} .
$$

A point in $\mathcal{A}$ gives a compatible family of vectors $v_{\mu} \in V_{\mu}$ for all highest weights $\mu$. A point in $(G / U)^{n}$ gives a vectors

$$
v_{\mu_{1}} \otimes v_{\mu_{2}} \otimes \cdots \otimes v_{\mu_{m}} \in V_{\mu_{1}} \otimes V_{\mu_{2}} \otimes \cdots \otimes V_{\mu_{m}}
$$

for all $m$-tuples of highest weights $\left(\mu_{1}, \mu_{2}, \ldots, \mu_{m}\right)$. Up to the action of $G$, we get a vector in

$$
\left[V_{\mu_{1}} \otimes V_{\mu_{2}} \otimes \cdots \otimes V_{\mu_{m}}\right]^{G} .
$$

Then the function $f$ evaluated on a point in $\operatorname{Conf}_{m}(\mathcal{A})$ is then given by contracting $f$ with the vector

$$
v_{\lambda_{1}} \otimes v_{\lambda_{2}} \otimes \cdots \otimes v_{\lambda_{m}}
$$

Let us now describe how to tropicalize $f$. A point in the affine Grassmannian of $G$ gives us not only a lattice, but a lattice in every representation of $G$. Let $x_{1}, \ldots, x_{m}$ be a configuration of points in the affine Grassmannian. For each $x_{i}$, we have a lattice $L_{i} \subset V_{\lambda_{i}} \otimes \mathcal{K}$. We maximize the expression

$$
-\operatorname{val}\left(f\left(v_{1}, v_{2}, \ldots, v_{m}\right)\right)
$$

over vectors $v_{i} \in L_{i}$. This maximum value will give $f^{t}\left(x_{1}, x_{2}, \ldots, x_{m}\right)$. 
6.2. Some EXAmples. Now, let us first make an easy observation. Because the outer automorphism of $S L_{n}$ acts on everything in sight, we have a dual statement to Theorem 5.10. Note that the outer automorphism of $S L_{n}$ takes any representation to its dual representation, and hence interchanges the weights $\omega_{a}$ and $\omega_{n-a}$. Now suppose that $i_{1}+i_{2}+\cdots+i_{k}=(k-1) n$. Then if we put $j_{s}=n-i_{s}$, we have that $j_{1}+j_{2}+\cdots+j_{k}=n$. We can then define the function

$$
f_{i_{1} i_{2} \ldots i_{k}}
$$

which is dual to the function $f_{j_{1} j_{2} \ldots j_{k}}$. These functions also turn out to be cluster variables $[4,8]$.

For points $x_{1}, x_{2}, \ldots, x_{k}$ in the affine building for $P G L_{n}$ or $S L_{n}$, we can define the functions

$$
f_{i_{1} i_{2} \ldots i_{k}}^{t}\left(x_{1}, x_{2} \ldots, x_{k}\right)
$$

by the procedure given in the previous section. Then we have that

THEOREM 6.1.

$$
f_{i_{1} i_{2} \ldots i_{k}}^{t}\left(x_{1}, x_{2} \ldots, x_{k}\right)=\min _{p}\left[d_{i_{1}}\left(p, x_{1}\right)+d_{i_{2}}\left(p, x_{2}\right)+\cdots+d_{i_{k}}\left(p, x_{k}\right)\right]
$$

where the minimum is taken over all $p$ in the affine Grassmannian for $P G L_{n}$.

This is an easy theorem given the above discussion, but it motivates the first example that goes beyond the results of this paper.

We will consider a function in the cluster algebra for $\operatorname{Conf}_{4}(\mathcal{A})$ constructed in [8]. Let $1 \leqslant a, b, c, d<n$ be four integers satisfying $a+b>n$ and $a+b+c+d=2 n$. Then there an invariants inside

$$
\left[V_{\omega_{a}} \otimes V_{\omega_{b}} \otimes V_{\omega_{c}} \otimes V_{\omega_{d}}\right]^{S L_{n}}
$$

given by the web in below.

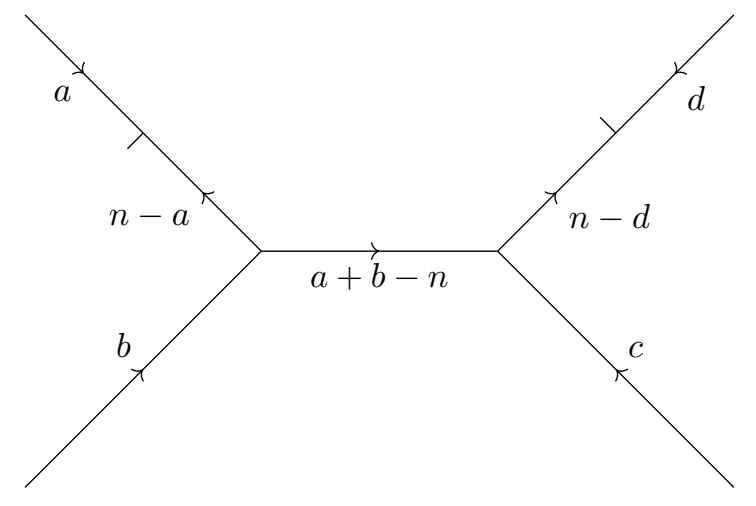

Here is a more concrete description of the function. Given four flags

first consider the forms

$$
\begin{gathered}
t_{1}, \ldots, t_{n} \\
u_{1}, \ldots, u_{n} \\
v_{1}, \ldots, v_{n} \\
w_{1}, \ldots, w_{n}
\end{gathered}
$$

$$
\begin{aligned}
T_{a} & :=t_{1} \wedge \cdots \wedge t_{a}, \\
U_{b} & :=u_{1} \wedge \cdots \wedge u_{b}, \\
V_{c} & :=v_{1} \wedge \cdots \wedge v_{c}, \\
W_{d} & :=w_{1} \wedge \cdots \wedge w_{d} .
\end{aligned}
$$


There is a natural map

$$
\phi_{a+b-n, n-a}: \bigwedge^{b} V \rightarrow \bigwedge^{a+b-n} V \otimes \bigwedge^{n-a} V .
$$

There are also natural maps

$$
W_{d} \wedge-\wedge V_{c}: \bigwedge^{a+c-n} V \rightarrow \bigwedge^{n} V \simeq F
$$

and

$$
T_{a} \wedge-: \bigwedge^{n-a} V \rightarrow \bigwedge^{n} V \simeq F
$$

Applying these maps to the first and second factors of $\phi_{a+c-n, n-a}\left(U_{b}\right)$, respectively, and then multiplying, we get get the value of our function. This is a function on $\mathrm{Conf}_{4} \mathcal{A}_{S L_{n}}$. Let us call this function $F$.

Conjecture 6.2. $F^{t}\left(x_{1}, x_{2}, x_{3}, x_{4}\right)$ is given by the minimum value of

$$
d_{a}\left(p, x_{1}\right)+d_{b}\left(p, x_{2}\right)+d_{a+b-n}(q, p)+d_{c}\left(q, x_{3}\right)+d_{d}\left(q, x_{4}\right)
$$

over $p$ and $q$ in the affine building for $P G L_{n}$.

In other words, the function $F^{t}$ is given by the minimal weighted distance over a graph embedded in the building. The leaves of the graph are prescribed to land on the points $x_{1}, x_{2}, x_{3}, x_{4}$, while the weights are determined by the web calculating the function $F$.

Acknowledgements. Two anonymous referees gave incisive and constructive feedback, which has greatly improved the exposition of this paper. I am grateful to Po-Shen Loh and Aaron Pixton for past discussions about the problems discussed in this paper, to Chris Fraser for his comments on a draft of this paper, and to Jim Geelen and Kazuo Murota noting the resemblance of the theorems in this paper to those in discrete convex analysis. Finally, I thank Evan O'Dorney for our collaboration [9], from which many of the ideas in this paper originated.

\section{REFERENCES}

[1] Vladimir Fock and Alexander Goncharov, Moduli spaces of local systems and higher Teichmüller theory, Publ. Math. Inst. Hautes Études Sci. (2006), no. 103, 1-211.

[2] Sergey Fomin, Michael Shapiro, and Dylan Thurston, Cluster algebras and triangulated surfaces. I. Cluster complexes, Acta Math. 201 (2008), no. 1, 83-146.

[3] Alexander Goncharov and Linhui Shen, Geometry of canonical bases and mirror symmetry, Invent. Math. 202 (2015), no. 2, 487-633.

[4] Donaldson-Thomas transformations of moduli spaces of G-local systems, Adv. Math. 327 (2018), 225-348.

[5] Mark Gross, Paul Hacking, Sean Keel, and Maxim Kontsevich, Canonical bases for cluster algebras, J. Amer. Math. Soc. 31 (2018), no. 2, 497-608.

[6] Joel Kamnitzer, Hives and the fibres of the convolution morphism, Selecta Math. (N.S.) 13 (2007), no. 3, 483-496.

[7] Ian Le, Higher laminations and affine buildings, Geom. Topol. 20 (2016), no. 3, 1673-1735.

[8] _ Cluster structures on higher Teichmuller spaces for classical groups, Forum Math. Sigma 7 (2019), Paper no. e13 (165 pages).

[9] Ian Le and Evan O'Dorney, Geometry of positive configurations in affine buildings, Doc. Math. 22 (2017), 1519-1538.

[10] Andrey G. Moshonkin, Concerning Hall's theorem, in Mathematics in St. Petersburg, Amer. Math. Soc. Transl. Ser. 2, vol. 174, Amer. Math. Soc., Providence, RI, 1996, pp. 73-77.

[11] Kazuo Murota, Discrete convex analysis, Math. Programming 83 (1998), no. 3, Ser. A, 313-371.

[12] Richard Rado, A theorem on independence relations, Quart. J. Math. Oxford Ser. 13 (1942), $83-89$.

IAN LE, Perimeter Institute for Theoretical Physics, Waterloo, ON N2L 2Y5, Canada

E-mail : ile@perimeterinstitute.ca 